\title{
Semiclassical approach to plasmon-electron coupling and Landau damping of surface plasmons
}

\author{
Yi Gao, ${ }^{1,2}$ Zhe Yuan, ${ }^{3}$ and Shiwu Gao ${ }^{2, a)}$ \\ ${ }^{1}$ Institute of Physics, Chinese Academy of Sciences, 10190 Beijing, China \\ ${ }^{2}$ Department of Physics, University of Gothenburg, SE-41296 Göteborg, Sweden \\ ${ }^{3}$ Department of Applied Physics, Chalmers University of Technology, SE-41296 Göteborg, Sweden
}

(Received 16 January 2011; accepted 15 March 2011; published online 5 April 2011)

\begin{abstract}
A semiclassical model is developed to describe plasmon-electron coupling and electronic damping of surface plasmons. It is compared with the ab initio linear response calculations for metallic thin films in the jellium approximation and for a realistic crystalline $\operatorname{Mg}(0001)$ surface. The semiclassical model is able to reproduce the quantum oscillations of plasmon linewidth, which was obtained in the previous ab initio calculations. In addition, state-resolved analysis reveals the origin of these oscillations, which result from superposition of the short-period oscillations of individual electron-hole pair transitions. The semiclassical model is further applied to a crystalline $\operatorname{Mg}(0001)$ surface, where linewidth dispersion of the surface plasmon is calculated and shows good agreement with earlier $a b$ initio calculation and experiment. Our results suggest that this semiclassical approach is quite promising for the quantitative description of plasmon-electron coupling and associated processes such as surface-enhanced Raman scattering, light emission, and fluorescence. () 2011 American Institute of Physics. [doi:10.1063/1.3575185]
\end{abstract}

\section{INTRODUCTION}

Many optical and electronic phenomena at metal surfaces $^{1-3}$ and in nanostructures ${ }^{4-11}$ are dominated by the collective excitations of the conduction electrons, namely, the surface plasmon. How long can such oscillations be sustained and how these oscillations decay at surfaces determine the outcome and efficiencies of various dynamical processes. For example, it is known that lifetime of surface plasmons sensitively affects the field enhancement in surface-enhanced Raman spectroscopy (SERS) and fluorescence. At solid surfaces, the lifetime of surface plasmon at finite momentum transfer is dominated by Landau damping, i.e., the decay of surface plasmons into electron-hole $(\mathrm{e}-\mathrm{h})$ pairs. Although the energy dispersions of surface plasmons can often be described by classical electrodynamics, ${ }^{6,7}$ the damping of surface plasmons is purely a quantum mechanical process and is governed by the coupling between the surface plasmons and $\mathrm{e}-\mathrm{h}$ pairs. This process is unfortunately not well understood even for the simplest crystalline surfaces.

So far, quantitative and systematic measurement of plasmon lifetime (or the linewidth) has been carried out mostly in metal thin films and overlayers. ${ }^{12-18}$ These measurements indicate that the plasmon linewidths depend sensitively on the interface structures and film thicknesses. For example, the plasmon linewidth of $\mathrm{Ag}$ films deposited on a $\mathrm{Cu}(111)$ surface changes by more than an order of magnitude when the film thickness is increased from 2 to 22 monolayers (ML), while the plasmon energy varies only by less than $10 \% .{ }^{15}$ Furthermore, this thickness dependence is nonmonotonic, but shows drastic oscillations as a function of thickness. ${ }^{13}$ Similar quantum oscillations in Landau damping have been observed in

\footnotetext{
a)Electronic mail: shiwu.gao@ physics.gu.se.
}

metal nanoparticles ${ }^{19-22}$ and thin films..$^{23,24}$ However, the oscillation period for thin films $1.7 \sim 2.0 \lambda_{\mathrm{F}}$, where $\lambda_{\mathrm{F}}$ is the Fermi wavelength of electrons, is much longer than that in the nanoparticles. It is also about three times longer than the universal period $\lambda_{\mathrm{F}} / 2$ observed in many properties of metal thin films. ${ }^{25-30}$ In the previous study, this oscillation period was qualitatively associated with the dynamical Friedel oscillation of the bulk electron gas. ${ }^{31}$ This explanation is questionable because electrons in the thin films are not bulk-like due to the confinement perpendicular to the films. The origin of such long-period oscillations and its difference from that of nanoparticles are still unknown. In particular, it remains unclear whether and how this oscillation is connected to the quantum well states (QWS) normal to the films.

Theoretically, plasmon-electron coupling (PEC) and electronic damping have usually been described by the following two different approaches. (1) One approach is ab intio calculations based on the linear response time-dependent local density approximations (LR-TDLDA), where the linewidth of the surface plasmon can be extracted from the lineshapes of the loss functions. The extracted linewidth measures the damping rate of surface plasmon by all electrons in the system. However, it gives no information about how the plasmon excitations couple with and decay into individual e-h pairs. ${ }^{35}$ Such an approach lacks physical transparency and cannot be applied to processes that require specific coupling between electrons and plasmon, for example, SERS. In addition, application of LR-TDLDA has been limited to a few simple and jellium surfaces ${ }^{32-34}$ and small nanostructures ${ }^{36,37}$ due to its high computational cost. (2) In contrast, model Hamiltonian of PEC (Refs. 20-22) has been developed in the literature. This approach is able to specify explicitly the coupling between surface plasmons with each and every electron-hole pairs. It also avoids the time-consuming computations of 
the many-body response functions. Such a semiclassical approach has been used for the description of plasmon damping of the Mie resonances in metallic nanoparticles. ${ }^{20-22}$ It is thus interesting to find out whether this approach can be extended to surfaces, thin films, and other geometries.

This paper presents a semiclassical model of plasmonelectron coupling and Landau damping for metal thin films and surfaces, based on the quantization of the plasmon hybridization (PH) model of Nordlander and co-workers. It is compared and calibrated with $a b$ initio LR-TDLDA calculations in the jellium model of thin metal films and a crystalline $\mathrm{Mg}(0001)$ surface. The semiclassical model is able to reproduce the oscillations of plasmon linewidth, which was obtained in the earlier LR-TDLDA calculations. Furthermore, state-resolved analysis from the semiclassical model reveals the origin of these oscillations, which result from superposition of the short-period oscillations in individual $\mathrm{e}-\mathrm{h}$ pair transitions. The semiclassical model is further applied to a crystalline $\mathrm{Mg}(0001)$ surface, where the linewidth dispersion of surface plasmon is calculated and shows good agreement with earlier $a b$ initio calculation and experiment. Our results suggest that the semiclassical approach is quite promising for the quantitative description of plasmon-electron coupling and associated processes such as surface-enhanced Raman scattering, light emission, and fluorescence in quasi-2D systems.

\section{THE SEMICLASSICAL MODEL}

Semiclassical models of PEC are usually based on quantization of the classical plasmon fields. ${ }^{38,39}$ Our model is obtained by quantization of the $\mathrm{PH}$ model of Nordlander and co-workers. ${ }^{6,7,40}$ From the PH model, the Hamiltonian of the surface plasmon of thin films ${ }^{40}$ can be written as

$$
\begin{aligned}
H= & \frac{n_{0} m_{\mathrm{e}}}{2 A} \sum_{s= \pm} \sum_{\mathbf{q}_{\|}} q_{\|}\left(1-e^{-2 q_{\|} D}\right) \\
& \times\left\{\dot{M}_{\mathbf{q}_{\| s}}^{*} \dot{M}_{\mathbf{q}_{\| s}}+\omega_{\mathbf{q}_{\| s} s}^{2} M_{\mathbf{q}_{\|} s}^{*} M_{\mathbf{q}_{\|} s}\right\}
\end{aligned}
$$

where $n_{0}$ is the electron density, $m_{\mathrm{e}}$ is the electron mass, $D$ is the thickness, and $A$ is the normalization area of the film. This Hamiltonian consists of two branches of surface plasmons, a low-energy symmetric mode (-) and a high-energy antisymmetric mode $(+)$, which result from hybridization between the surface plasmons of the two surfaces. ${ }^{6,7}$ The dispersions are given as $\omega_{\mathbf{q}_{\|} \pm}=\omega_{\text {sp }}\left(1 \pm e^{-q_{\|} D}\right)^{1 / 2}$, and $\omega_{\text {sp }}$ is the surface plasmon frequency. The collective coordinates with momentum transfer $\mathbf{q}_{\|}$of the two modes, $M_{\mathbf{q}_{\|} \pm}$, can be transformed into

$$
\mathcal{M}_{\mathbf{q}_{\|} \pm}=\sqrt{\frac{\hbar}{2 q_{\|} n_{0} m_{\mathrm{e}} \omega_{\mathbf{q}_{\|} \pm}\left(1-e^{-2 q_{\|} D}\right)}}\left(a_{\mathbf{q}_{\|} \pm}+a_{-\mathbf{q}_{\|} \pm}^{\dagger}\right) .
$$

From the quantization condition for the annihilation $\left(a_{\mathbf{q}_{\|} \pm}\right)$ and creation operator $\left(a_{\mathbf{q}_{\|} \pm}^{\dagger}\right)$,

$$
\left[a_{\mathbf{q}_{\|} \pm}, a_{\mathbf{q}_{\|}^{\prime} \pm}^{\dagger}\right]=\delta_{\mathbf{q}_{\|} \mathbf{q}_{\|}^{\prime}}
$$

the Hamiltonian of Eq. (1) is now quantized as

$$
\mathcal{H}=\frac{1}{A} \sum_{s= \pm} \sum_{\mathbf{q}_{\|}} \hbar \omega_{\mathbf{q}_{\|} s}\left(a_{\mathbf{q}_{\| s}}^{\dagger} a_{\mathbf{q}_{\|} s}+\frac{1}{2}\right)
$$

The induced potential of the classical plasmon field, ${ }^{40}$

$$
\begin{aligned}
\delta V_{ \pm}(\mathbf{x})= & \frac{2 \pi n_{0} e_{0}^{2}}{\sqrt{2 A}} \sum_{\mathbf{q}_{\|}} M_{\mathbf{q}_{\|} \pm}\left(1 \pm e^{-q_{\|} D}\right) \\
& \times\left(e^{-q_{\|}|z|} \mp e^{-q_{\|}|z+D|}\right) e^{i \mathbf{q}_{\|} \cdot \mathbf{x}_{\|}},
\end{aligned}
$$

can also be quantized accordingly:

$$
\delta \mathcal{V}_{ \pm}(\mathbf{x})=\sum_{\mathbf{q}_{\|}} v_{ \pm}\left(q_{\|}, z\right)\left(a_{\mathbf{q}_{\|} \pm}+a_{-\mathbf{q}_{\|} \pm}^{\dagger}\right) e^{i \mathbf{q}_{\|} \cdot \mathbf{x}_{\|}}
$$

where $\quad v_{ \pm}\left(q_{\|}, z\right)=e_{0} \sqrt{\left[\pi \hbar \omega_{\mathbf{q}_{\|} \pm} / 2 q_{\|} A\left(1 \mp e^{-q_{\|} D}\right)\right]}\left(e^{-q_{\|}|z|}\right.$ $\mp e^{-q_{\|}|z+D|}$ ), and $e_{0}$ is the electron charge. This potential has the same spatial dependence as its classical form in Eq. (5) but has now attached a quantum amplitude after quantization.

The damping rate for the surface plasmons is calculated from Fermi's golden rule as

$$
\begin{aligned}
\Gamma\left(\mathbf{q}_{\|}, \omega_{\mathbf{q}_{\|} \pm}\right)= & \frac{2 \pi}{\hbar} \sum_{\mathbf{k}_{\|}} \sum_{n, n^{\prime}} f_{n \mathbf{k}_{\|}}\left(1-f_{n^{\prime} \mathbf{k}_{\|}+\mathbf{q}_{\|}}\right) \\
& \times \mid\left\langle n^{\prime} \mathbf{k}_{\|}+\mathbf{q}_{\|}, m_{\mathbf{q}_{\|} \pm}=0\right| \\
& \times\left. v_{ \pm}\left(q_{\|}, z\right) a_{\mathbf{q}_{\|} \pm} e^{i \mathbf{q}_{\|} \cdot \mathbf{x}_{\|}}\left|n \mathbf{k}_{\|}, m_{\mathbf{q}_{\|} \pm}=1\right\rangle\right|^{2} \\
& \times \delta\left(\hbar \omega_{\mathbf{q}_{\|} \pm}+\varepsilon_{n \mathbf{k}_{\|}}-\varepsilon_{n^{\prime} \mathbf{k}_{\|}+\mathbf{q}_{\|}}\right)
\end{aligned}
$$

where $m_{\mathbf{q}_{\|} \pm}$are quantum numbers of plasmons, and $\left|n \mathbf{k}_{\|}\right\rangle$ $\left(\left|n^{\prime} \mathbf{k}_{\|}+\mathbf{q}_{\|}\right\rangle\right)$is electron (hole) state with eigenenergy $\varepsilon_{n \mathbf{k}_{\|}}$ $\left(\varepsilon_{n^{\prime}} \mathbf{k}_{\|}+\mathbf{q}_{\|}\right)$and occupation number $f_{n \mathbf{k}_{\|}}\left(f_{n^{\prime} \mathbf{k}_{\|}+\mathbf{q}_{\|}}\right)$. The Landau damping rate given by Eq. (7) shows that it is determined by two major ingredients: (i) the coupling potential, which is specified in Eq. (6), and (ii) the eigenenergies and wavefunctions of the $\mathrm{e}-\mathrm{h}$ pairs involved in the damping. The latter can be found in previous publications. ${ }^{23,24}$

Plasmon-electron coupling of a semi-infinite surface can be simply obtained from the asymptotic limit $D \rightarrow \infty$ of the thin film model. In this limit, $\omega_{\mathbf{q}_{\|} \pm}=\omega_{\text {sp }}$, and the coupling potential $v\left(q_{\|}, z\right)=e_{0} \sqrt{\left(\pi \hbar \omega_{\mathrm{sp}} / q_{\|} A\right)} e^{-q_{\|}|z|}$. The $z=0$ plane is chosen at the dynamical image plane obtained from LRTDLDA, ${ }^{2,23}$ where the plasmon induced density and potential are peaked. The damping rate of surface plasmon is thus

$$
\begin{aligned}
\Gamma\left(\mathbf{q}_{\|}, \omega_{\mathrm{sp}}\right)= & \frac{2 \pi}{\hbar} \sum_{\mathbf{k}_{\|}} \sum_{k_{z}, k_{z}^{\prime}} f_{k_{z} \mathbf{k}_{\|}}\left(1-f_{k_{z}^{\prime} \mathbf{k}_{\|}+\mathbf{q}_{\|}}\right) \\
& \times \mid\left\langle k_{z}^{\prime} \mathbf{k}_{\|}+\mathbf{q}_{\|}, m_{\mathbf{q}_{\|}}=0\right| v\left(q_{\|}, z\right) \\
& \times\left. a_{\mathbf{q}_{\|}} e^{i \mathbf{q}_{\|} \cdot \mathbf{x}_{\|}}\left|k_{z} \mathbf{k}_{\|}, m_{\mathbf{q}_{\|}}=1\right\rangle\right|^{2} \\
& \times \delta\left(\hbar \omega_{\mathrm{sp}}+\varepsilon_{k_{z} \mathbf{k}_{\|}}-\varepsilon_{k_{z}^{\prime} \mathbf{k}_{\|}+\mathbf{q}_{\|}}\right) .
\end{aligned}
$$




\section{RESULTS AND DISCUSSION}

\section{A. Landau damping of surface plasmon in thin films}

To find out how the semiclassical PEC model works for thin films, we calculated the Landau damping of surface plasmon in the $r_{\mathrm{s}}=3$ jellium slab with variable thicknesses. This system was studied in early publications ${ }^{23,24}$ using the LRTDLDA approach and is now used as a model system for calibration of the semiclassical model. Metallic thin films are described by the jellium model as in the previous studies. ${ }^{23,24}$ The electron density of $r_{\mathrm{s}}=3$ with Fermi wavelength 9.82 a.u. is chosen to model silver without considering its $d$ electrons. Inclusion of background polarization should lead to significant redshift of plasmon energies. This is, however, not the focus of this work. A vacuum layer of $12 \lambda_{\mathrm{F}}\left(6 \lambda_{\mathrm{F}}\right.$ on each side) was found to give enough number of scattering states, ${ }^{23}$ yielding converged loss spectra in LR-TDLDA and the semiclassical linewidth. A broadening parameter of $27 \mathrm{meV}$ is used in the calculation of $\chi_{0}$ to avoid divergence and to speed up the calculation. This parameter is much smaller than the physical linewidths extracted from the response functions and does not affect the results.

Figure 1 shows the eigenenergies of the QWS normal to the films. These QWS together with the 2D free-electron bands form the 3D band structures and wavefunctions of the thin films. The eigenenergies are shown as a function of thickness in unit of $\lambda_{F}$. The zero of the energy is referred to the vacuum level. The shaded area marks the occupied states, while the upper part is the unoccupied states. These spectra were generated in the same way as in previous calculations. ${ }^{23,24}$ It is clear that the Fermi level (or the work function) oscillates with a period of $\lambda_{F} / 2$. This oscillation results from the quantum size effect of the thin films and is generally responsible for the quantum oscillations in work function, electron-

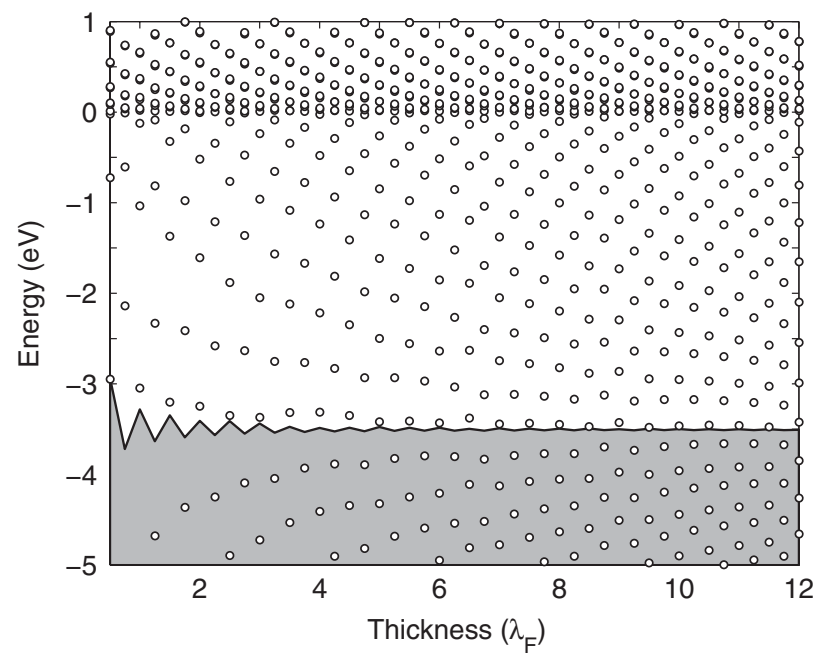

FIG. 1. Calculated eigenenergies of QWS in metallic films with $r_{\mathrm{s}}=3.0$ around the Fermi level. A vacuum region of $12 \lambda_{\mathrm{F}}$ is used for all thicknesses. The shaded area indicates the occupied states, whose Fermi energy oscillates with a period of $\lambda_{\mathrm{F}} / 2$. The discretized scattering states above the vacuum level (at zero) are also displayed. These continuum states are generated simultaneously as the bound states.

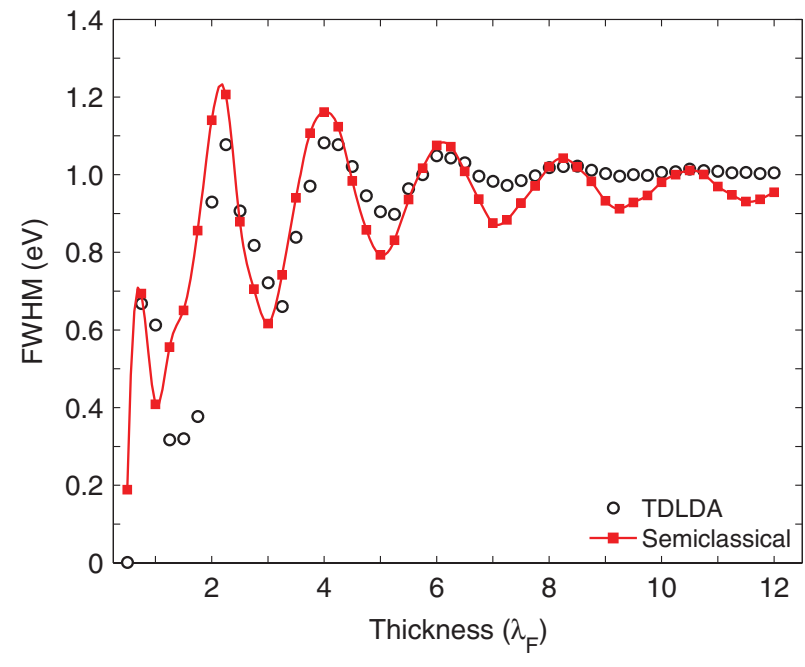

FIG. 2. Full width at half maximum of the symmetric surface plasmon mode as a function of film thickness at $q_{\|}=0.10$ a.u. calculated by TDLDA (open circles) and the plasmon-electron coupling model (squared line).

phonon coupling, and transition temperatures of superconductivity observed in various experiments.

We focus on the damping rate of symmetric branch only, although calculations and comparison have also been done for the antisymmetric mode. Figure 2 shows the full width at half maximum (FWHM) of the symmetric plasmon mode extracted from LR-TDLDA and from the semiclassical model given by Eq. (7). Here the semiclassical linewidth is defined as $\hbar \Gamma$. The two linewidths are in good agreement at all thicknesses that were calculated. Both linewidths oscillate with the thickness, although the oscillations are more drastic in the semiclassical model at large thicknesses. In the semiclassical calculation, the energy dispersion of the symmetric plasmon is taken from the classical expression, which is close to the dispersion given by LR-TDLDA. ${ }^{23,24}$ Test calculations have also been done for the FWHM at other $q_{\|}$values for both symmetric and antisymmetric modes, which are all in good agreement with the LR-TDLDA results. It should be noted that the computations by the PEC model involve only the band structures and wavefunctions of the ground state, and avoids the linear response calculations, which is computationally much more demanding. The good agreement between the PEC model and LR-TDLDA indicates that the PEC model captures the essential physics of plasmon-electron coupling and gives reliable damping rate of surface plasmons. It is therefore possible and appealing to apply this model to describe plasmon-electron coupling in larger systems and more realistic structures in the future.

In order to gain insight into the quantum oscillations shown in Fig. 2, especially its large period, we carried out a state-resolved analysis of the damping rate using the semiclassical model as shown in Fig. 3. This is only possible in the semiclassical model, where the damping rate is calculated by the golden-rule expression. And contributions from each initial state (subband) can be calculated and analyzed separately. The total FWHM for $q_{\|}=0.1$ a.u. is again given as the squared line, which has an oscillation period of about $2 \lambda_{\mathrm{F}}$. The partial damping rate corresponding to transitions from 


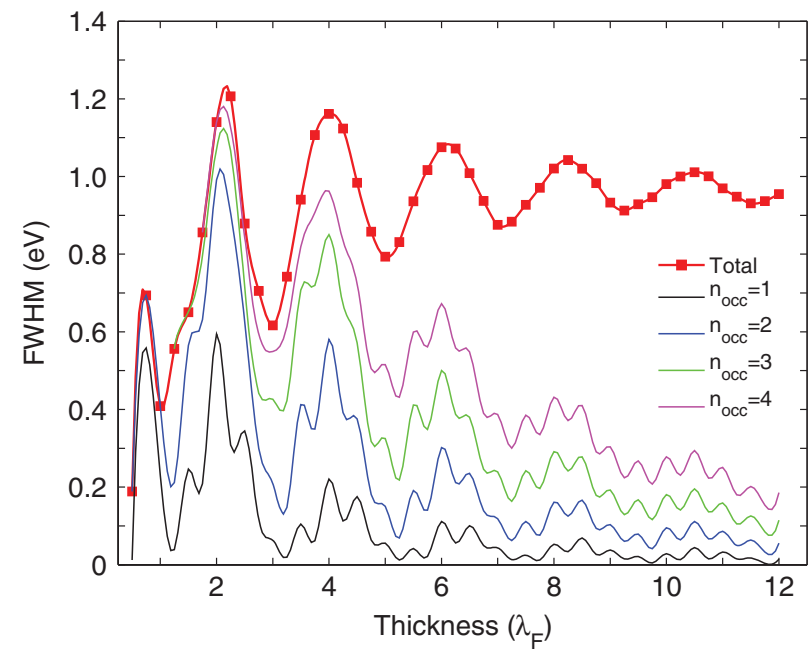

FIG. 3. Damping rates calculated by partial summation of different initial states including successively the HOMO state $\left(n_{\text {occ }}=1\right)$, HOMO- $1\left(n_{\text {occ }}\right.$ $=2)$, HOMO-2 $\left(n_{\mathrm{occ}}=3\right)$, HOMO-3 ( $\left.n_{\mathrm{occ}}=4\right)$, and HOMO-4 $\left(n_{\mathrm{occ}}=5\right)$ of the quantum well states below the Fermi levels. These states contribute dominantly to the total width (squared line). Superposition of individual transitions gradually destroys the $\lambda_{\mathrm{F}} / 2$ oscillation period associated with single particle excitations, and gives rise to a large period of about $1.7 \sim 2.0 \lambda_{\mathrm{F}}$ that is characteristic for the dynamical Friedel oscillations.

the highest occupied state (HOMO) is given by the bottom line in the figure $\left(n_{\mathrm{occ}}=1\right)$. This single-band rate oscillates with a period of $\lambda_{\mathrm{F}} / 2$, the universal period observed in work function and other quantities. Obviously, this short period is a signature of single-particle effect. Including one more band beneath the HOMO-1 state, the partial damping rate is shown as the second line from the bottom $\left(n_{\text {occ }}=2\right)$. Short-period oscillations are still visible at large thicknesses. However, at small thicknesses, this partial rate is almost comparable to the total damping rate for up to $D=2.5 \lambda_{\mathrm{F}}$. In this regime, superposition of the two bands almost destroys the $\lambda_{\mathrm{F}} / 2$-period oscillations. As a result, oscillations with a large period start to emerge. Including more occupied states into the damping rate $\left(n_{\text {occ }}=3,4\right)$, more oscillations with the large period appear at increasing thicknesses. This analysis clearly shows transformation from individual e-h-like excitations with a period of $\lambda_{F} / 2$ to the collective oscillation, which has a period of about $2 \lambda_{\mathrm{F}}$. It also demonstrates how collective behavior develops from the superposition of individual $\mathrm{e}-\mathrm{h}$ pair excitations. The analysis in Fig. 3 reveals the origin of the linewidth oscillations with the film thickness in a much more transparent manner.

\section{B. Damping of surface plasmon at $\mathrm{Mg}(0001)$ surface}

Next we apply the semiclassical model to a realistic crystalline $\operatorname{Mg}(0001)$ surface, where the energy dispersion and the damping rate of the surface plasmons have been measured experimentally. It serves as a prototype system for testing the PEC model in extended systems beyond the jellium approximation.

The $\operatorname{Mg}(0001)$ surface is modeled by a slab of 28 layers of $\mathrm{Mg}$ atoms. The lattice constants are chosen from experimental data, i.e., $a=3.21 \AA$ and $c=5.21 \AA$. A supercell with a vacuum region corresponding to $8 \mathrm{ML}$ of $\mathrm{Mg}$ atoms is included in the ground state discrete Fourier transform calculation. The Troullier-Martins pseudopotential ${ }^{41}$ is used for Mg. The wavefunctions are expanded in plane waves with an energy cutoff at $12 \mathrm{Ry}$. The Brillouin zone integration is performed by summation over a $90 \times 90 \times 1 k$-point
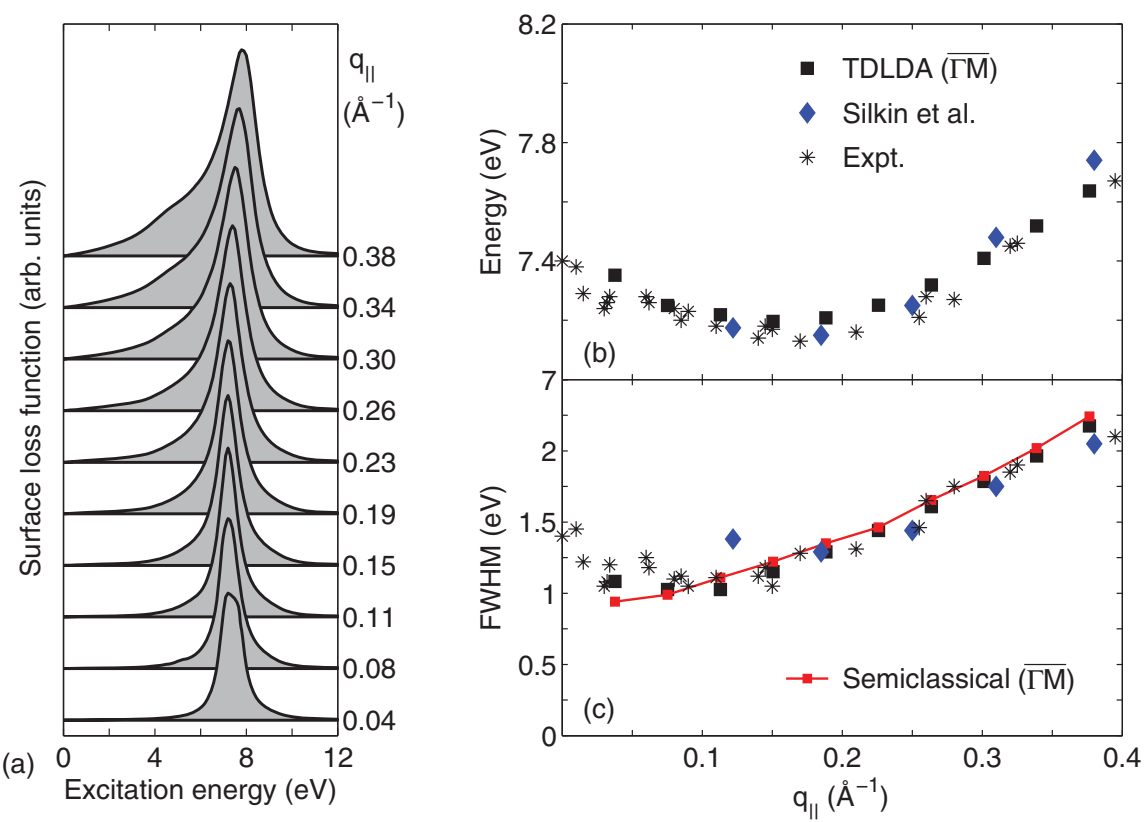

FIG. 4. (a) Surface loss function of $\operatorname{Mg}(0001)$ as a function of momentum transfer along $\overline{\Gamma M}$. The calculation was done in LR-TDLDA using a 28 ML magnesium slab. (b) Comparison of surface plasmon dispersions obtained by TDLDA (squares), early calculation by Silkin et al. (Ref. 33) (diamonds), and experimental data (Ref. 44). (c) FWHM dispersions obtained by TDLDA (squares), the model (squared line), and experimental data (Ref. 44). In the semiclassical model, we used the TDLDA plasmon dispersion in panel (b) and the dynamical image plane $z_{\text {dip }}=d\left(q_{\|}, \omega\right)$ (Refs. 24 and 2$)$ which is about 1 a.u. outside the surface, as the reference plane in the coupling potential. 
mesh, yielding high resolution of momentum transfer in linear response calculations. Response functions are calculated within the mixed momentum-coordinate scheme. ${ }^{34}$ Ceperley-Alder's local density functional ${ }^{42}$ parameterized by Perdew and Zunger ${ }^{43}$ is used throughout all calculations.

Silkin et al. have shown that band structure has to be taken into account in order to obtain reliable surface plasmon linewidths for $\operatorname{Mg}(0001)$ surface. ${ }^{33}$ Starting from the ground state band structure, we performed both LR-TDLDA and semiclassical calculations for the energy dispersion and the rate of Landau damping. The results are shown in Fig. 4. Panel (a) shows the surface loss spectra with momentum transfers along the $\overline{\Gamma M}$ direction of the surface Brillouin zone. The energy dispersion and FWHM linewidth are given in panels (b) and (c) as a function of momentum. The energy dispersions given by TDLDA are in good agreement with experimental data ${ }^{44}$ and the early calculations by Silkin and collaborators. The Landau damping rate in the semiclassical model is calculated by using the TDLDA plasmon dispersions found in panel (b). Using the classical energy dispersion does not change appreciably the damping rate. Once again, the FWHM given by the semiclassical model is in quite good agreement with that of TDLDA and experiment. Figure 4 shows that the PEC model is able to reproduce the damping rate of the surface plasmons on the crystalline surfaces.

\section{CONCLUSIONS}

In summary, we have presented a semiclassical model of plasmon-electron coupling and electronic damping of surface plasmons in the metallic thin films and the $\operatorname{Mg}(0001)$ surface with realistic band structures. The semiclassical PEC model successfully reproduces the $a b$ initio LR-TDLDA results. In the jellium approximation of the thin films, the linewidths of the surface plasmons show an oscillatory dependence on the film thickness with a period of $1.7 \sim 2 \lambda_{\mathrm{F}}$. State-resolved analysis by the PEC model revealed that this long-period oscillation results from the superposition of individual $\mathrm{e}-\mathrm{h}$ pair transitions. We further extended our study to crystalline $\operatorname{Mg}(0001)$ surface, where realistic band structure is taken into account. The damping rates given by the semiclassical model agree well with the $a b$ initio calculations and experimental data. This suggests that the semiclassical model captures the essential physics of plasmon-electron coupling of realistic metal systems, and offers a promising approach for the description of dynamical processes involving plasmon-electron coupling near surfaces of nanostructures.

\section{ACKNOWLEDGMENTS}

This work has been supported by the Outstanding Young Researcher program of NSFC, the 973 program of MOST, China, and the Institutional Grant Program of STINT, Sweden.
${ }^{1}$ M. Rocca, Surf. Sci. Rep. 22, 1 (1995).

${ }^{2}$ A. Liebsch, Electronic Excitations at Metal Surfaces (Plenum, New York, 1997).

${ }^{3}$ J. M.Pitarke, V. M. Silkin, E. V. Chulkov, and P. M. Echenique, Rep. Prog. Phys. 70, 1 (2007).

${ }^{4}$ S. A. Maier, Plasmonics-Fundamentals and Applications, 1st ed. (Springer, New York, 2007).

${ }^{5}$ H. X. Xu, E. J. Bjerneld, M. Käll, and L. Börjesson, Phys. Rev. Lett. 83, 4357 (1999).

${ }^{6}$ E. Prodan, C. Radloff, N. Halas, and P. Nordlander, Science 302, 419 (2003).

${ }^{7}$ E. Prodan and P. Nordlander, J. Chem. Phys. 120, 5444 (2004).

${ }^{8}$ S. Lal, S. Link, and N. J. Halas, Nat. Photonics 1, 641 (2007).

${ }^{9}$ D. Neuhauser and K. Lopata, J. Chem. Phys. 127, 154715 (2007).

${ }^{10}$ K. Lopata and D. Neuhauser, J. Chem. Phys. 130, 104707 (2009).

${ }^{11}$ J. Zuloaga, E. Prodan, and P. Nordlander, ACS Nano 4, 5269 (2010).

${ }^{12}$ F. Moresco, M. Rocca, T. Hildebrandt, and M. Henzler, Phys. Rev. Lett. 83, 2238 (1999).

${ }^{13}$ Y. H. Yu, Y. Jiang, Z. Tang, Q. L. Guo, J. F. Jia, Q. K. Xue, K. H. Wu, and E. G. Wang, Phys. Rev. B 72, 205405 (2005).

${ }^{14}$ A. Politano, R. G. Agostino, E. Colavita, V. Formoso, and G. Chiarello, J. Chem. Phys. 126, 244712 (2007).

${ }^{15}$ A. Politano, V. Formoso, E. Colavita, and G. Chiarello, Phys. Rev. B 79, 045426 (2009).

${ }^{16}$ A. Politano and G. Chiarello, Surf. Rev. Lett. 16, 171 (2008).

${ }^{17}$ A. Politano and G. Chiarello, Phys. Status Solidi (RRL) 3, 136 (2009).

${ }^{18}$ A. Politano, V. Formoso, and G. Chiarello, J. Nanosci. Nanotechnol. 10, 1313 (2010).

${ }^{19}$ C. Yannouleas and R. A. Broglia, Ann. Phys. (NY) 217, 105 (1992).

${ }^{20}$ R. A. Molina, D. Weinmann, and R. A. Jalabert, Phys. Rev. B 65, 155427 (2002)

${ }^{21}$ G. Weick, R. A. Molina, D. Weinmann, and R. A. Jalabert, Phys. Rev. B 72, 115410 (2005)

${ }^{22}$ E. Zaremba and B. N. J. Persson, Phys. Rev. B 35, 596 (1987).

${ }^{23}$ Z. Yuan and S. W. Gao, Surf. Sci. 602, 460 (2008).

${ }^{24}$ Z. Yuan and S. W. Gao, Phys. Rev. B 73, 155411 (2006).

${ }^{25}$ A. R. Smith, K.-J. Chao, Q. Niu, and C.-K. Shih, Science 273, 226 (1996).

${ }^{26}$ D.-A. Luh, T. Miller, J. J. Paggel, M. Y. Chou, and T.-C. Chiang, Science 292, 1131 (2001).

${ }^{27}$ Z. Y. Zhang, Q. Niu, and C.-K. Shih, Phys. Rev. Lett. 80, 5381 (1998).

${ }^{28}$ Y. Jia, B. Wu, H. H. Weitering, and Z. Y. Zhang, Phys. Rev. B 74, 035433 (2006).

${ }^{29}$ B. Wu and Z. Y. Zhang, Phys. Rev. B 77, 035410 (2008).

${ }^{30}$ Y. Jia, B. Wu, C. Li, T. L. Einstein, H. H. Weitering, and Z. Y. Zhang, Phys. Rev. Lett. 105, 066101 (2010).

${ }^{31}$ A. Liebsch, Phys. Rev. B 36, 7378 (1987).

${ }^{32}$ A. G. Eguiluz, Phys. Rev. Lett. 51, 1907 (1983).

${ }^{33}$ V. M. Silkin, E. V. Chulkov, and P. M. Echenique, Phys. Rev. Lett. 93, 176801 (2004).

${ }^{34}$ Z. Yuan and S. W. Gao, Comput. Phys. Commun. 180, 466 (2008).

${ }^{35}$ D. Bohm and D. Pines, Phys. Rev. 92, 609 (1953).

${ }^{36}$ E. Prodan, P. Nordlander, and N. J. Halas, Nano Lett. 3, 1411 (2003).

${ }^{37}$ C. L. Nehl, N. K. Grady, G. P. Goodrich, F. Tam, N. J. Halas, and J. H. Hafner, Nano Lett. 4, 2355 (2004).

${ }^{38}$ D. J. Bergman and M. I. Stockman, Phys. Rev. Lett. 90, 027402 (2003).

${ }^{39}$ A. Archambault, F. Marquier, and J.-J. Greffet, Phys. Rev. B 82, 035411 (2010)

${ }^{40}$ F. Le, N. Z. Lwin, N. J. Halas, and P. Nordlander, Phys. Rev. B 76, 165410 (2007)

${ }^{41}$ N. Troullier and J. L. Martins, Phys. Rev. B 43, 1993 (1991).

${ }^{42}$ D. M. Ceperley and B. J. Alder, Phys. Rev. Lett. 45, 566 (1980).

${ }^{43}$ J. P. Perdew and A. Zunger, Phys. Rev. B 23, 5048 (1981).

${ }^{44}$ P. D. Sprunger, G. M. Watson, and E. W. Plummer, Surf. Sci. 269/270, 551 (1992). 